\title{
Novel therapy for anti-glomerular basement membrane disease with IgA nephropathy: A case report
}

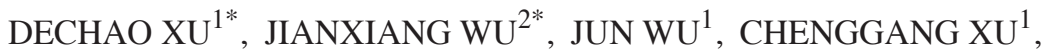 \\ YUQIANG ZHANG ${ }^{1}$, CHANGLIN MEI $^{1}$ and XIANG GAO ${ }^{1}$ \\ ${ }^{1}$ Department of Medicine, Kidney Institute of PLA, Changzheng Hospital, Second Military Medical University, \\ Shanghai 200003; ${ }^{2}$ Department of Geriatrics, No. 411 Hospital of PLA, Shanghai 200081, P.R. China
}

Received June 21, 2015; Accepted February 11, 2016

DOI: $10.3892 /$ etm.2016.3149

\begin{abstract}
Anti-glomerular basement membrane (GBM) disease is characterized by circulating anti-GBM antibodies and deposition of these antibodies in the renal GBM. Renal involvement in anti-GBM is more severe when compared with other types of immune-mediated glomerulonephritis, and the majority of patients manifest progressive renal failure, leading to end-stage renal disease. In a limited number of cases, anti-GBM disease has been shown to be accompanied with other immune-mediated glomerulonephritis. The present study reported the case of a 50-year-old female patient presenting with rapidly progressive glomerulonephritis, who was diagnosed with anti-GBM disease with IgA nephropathy. The patient achieved a relatively good therapeutic outcome with administration of corticosteroids plus mycophenolate mofetil (MMF), which may prove to be a novel treatment option for this rare disease; however, the exact underlying mechanism requires further in-depth investigation.
\end{abstract}

\section{Introduction}

Anti-glomerular basement membrane (anti-GBM) disease is a classic organ-specific autoimmune disease, mainly involving the lungs and kidneys. It is characterized by circulating anti-GBM antibodies and deposition of these antibodies in the renal GBM $(1,2)$. The incidence of anti-GBM disease is estimated to be 1 case per million per year, but it accounts for $20 \%$ of all cases of rapidly progressive or crescentic glomerulonephritis, and the age distribution is bimodal,

Correspondence to: Dr Xiang Gao, Department of Medicine, Kidney Institute of PLA, Changzheng Hospital, Second Military Medical University, 415 Fengyang Road, Shanghai 200003, P.R. China

E-mail: gaoxiang43100636@163.com

${ }^{*}$ Contributed equally

Key words: anti-glomerular basement membrane disease, immunoglobulin A nephropathy, mycophenolate mofetil
20-30 years old and 60-70 years old $(3,4)$. Renal pathological analysis in anti-GBM patients has previously demonstrated that immunoglobulin (Ig) $\mathrm{G}$ with or without C3 was linearly deposited in the basement membrane, while light microscopy mainly identified crescentic nephritis (5). Renal involvement in anti-GBM is more severe when compared with other types of immune-mediated glomerulonephritis. The majority of patients present progressive renal failure, resulting in end-stage renal disease, and require long-term treatment to improve the prognosis (3). According to the Kidney Disease - Improving Global Outcomes Clinical Practice Guideline for glomerulonephritis, corticosteroids and cyclophosphamide in combination with plasmapheresis are recommended as the initial immunosuppressive treatment for anti-GBM disease (6). The association between anti-GBM disease and antineutrophil cytoplasmic antibody-associated vasculitis has been well established in an increased number of studies (7-9). However, only a limited number of studies have reported the association between anti-GBM disease and $\operatorname{Ig}$ A nephropathy or other immune complex glomerulonephritis, such as Schönlein-Henoch purpura and membranous glomerulonephritis (10-13).

The present study reports the case of a patient who was diagnosed with anti-GBM disease with IgA nephropathy, and was successfully treated with corticosteroids in combination with mycophenolate mofetil (MMF).

\section{Case report}

A 50-year-old female was admitted to the Kidney Institute of PLA (Changzheng Hospital, Shanghai, China) in June 2014 with complaints of gross hematuria and rapidly decreasing kidney function for the past 2 weeks. The patient did not experience any fever, skin rash and hemoptysis. Gross hematuria was presented 2 weeks prior to admission, and laboratory tests performed in a local hospital reported hematuria with a red blood cell (RBC) count of 336 cells/ $\mu$ l (the normal level is $<25$ cells $/ \mu 1$ ), as well as decreased kidney function with a serum creatinine (Scr) level of $157 \mu \mathrm{mol} / 1$ (the normal level is 61-116 $\mu \mathrm{mol} / \mathrm{l}$ ) and blood urea nitrogen level of $11.29 \mathrm{mmol} / \mathrm{l}$ (the normal level is $2.9-7.2 \mathrm{mmol} / \mathrm{l}$ ). Ultrasonography reveal that the kidneys were normal in size and shape, with the exception of several small kidney stones. The diagnosis of renal lithiasis was established at the local hospital, without 
A

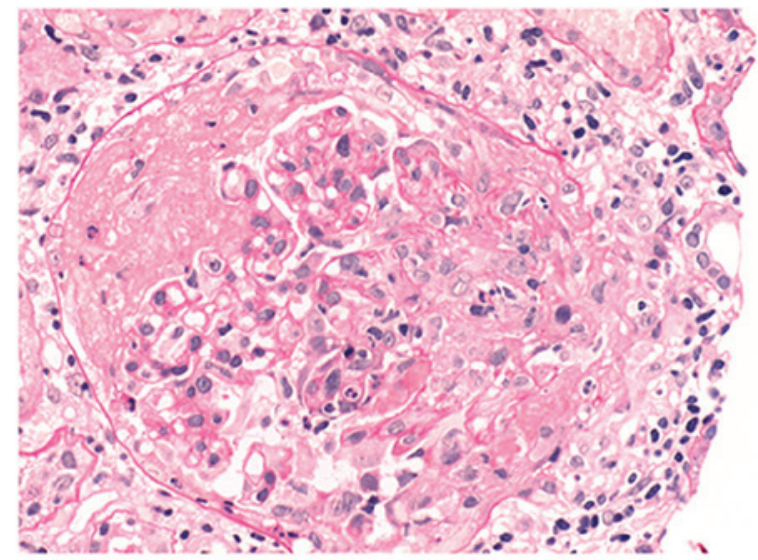

C

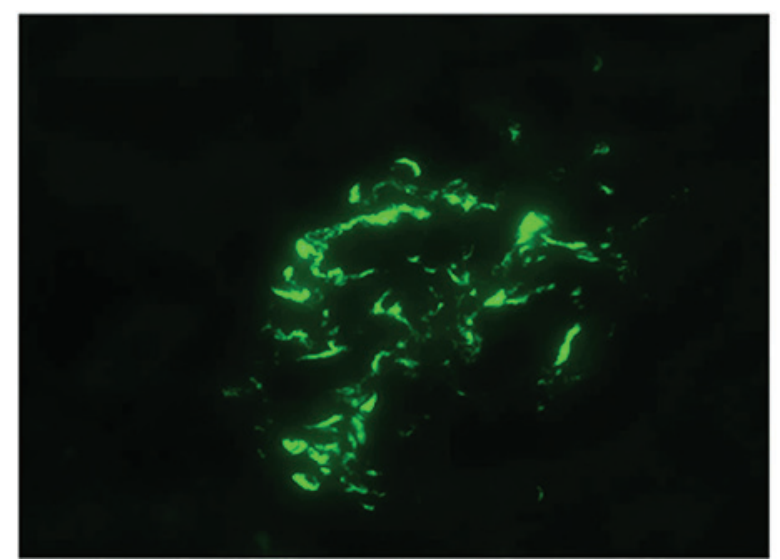

B

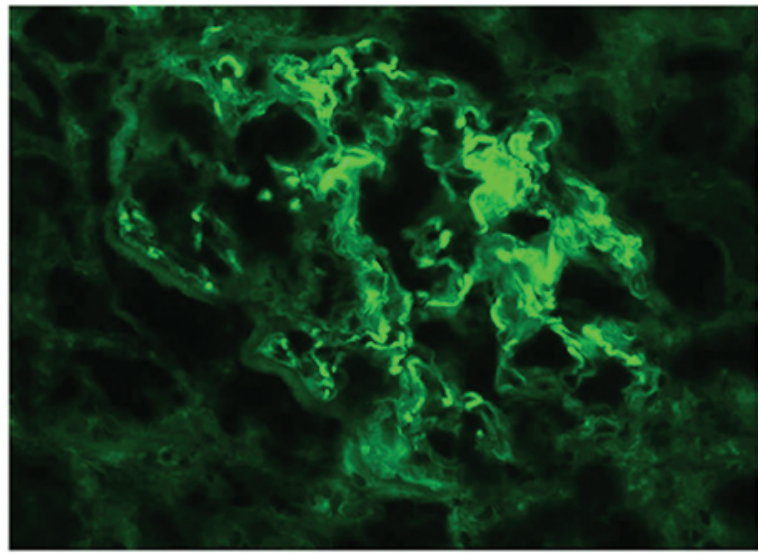

D

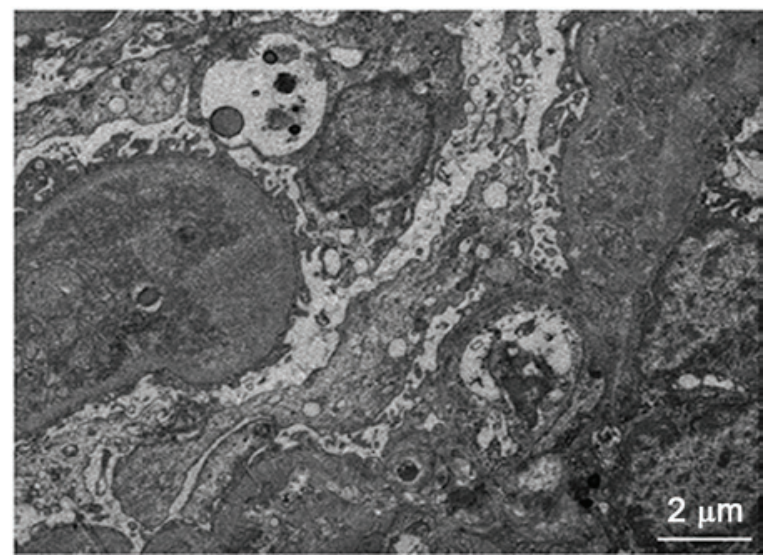

Figure 1. Pathology of renal biopsy. (A) Light microscopy showing hypercellularity with cellularofibrous crescent (PAS staining). (B) Immunofluorescence showing linear deposition of IgG along the GBM. (C) Immunofluorescence showing lumpy deposition of IgA in the mesangium. (D) Electron microscopy revealing electrondense deposition in the mesangium. Ig, immunoglobulin; GBM, glomerular basement membrane.

administration of any specific treatment. However, the patient was advised to drink more water and was prescribed a traditional Chinese medicine called Loosestrife (Guangxi Wantong Pharmaceutical Co., Ltd., Nanning, China). However, gross hematuria did not show any improvement 10 days later, and further laboratory tests suggested a rapidly decreasing kidney function with an Scr level of $220 \mu \mathrm{mol} / 1$.

Subsequently, the patient was transferred to Changzheng Hospital for further diagnosis and treatment. Physical examination was not remarkable. Laboratory examination upon admission included counting the number of RBCs per HP by observing the urine under a DM2500 HP microscope (Leica, Heidelberg, Germany). The results showed heavy hematuria [10-15 RBCs per high-power field (HP); the normal level is $<3$ RBCs per HP], with $90 \%$ dysmorphic RBCs, mild urinary protein excretion $(0.41 \mathrm{~g} / 24 \mathrm{~h}$; the normal level is $<0.15 \mathrm{~g} / 24 \mathrm{~h})$, and decreased kidney function with an Scr level of $232 \mu \mathrm{mol} / \mathrm{l}$. In addition, the hemoglobin ( $\mathrm{Hb}$ ) level was $99 \mathrm{~g} / 1$ (the normal level is $110-150 \mathrm{~g} / \mathrm{l}$ ) and the erythrocyte sedimentation rate (ESR) was $141 \mathrm{~mm} / \mathrm{h}$ (the normal level is $0-20 \mathrm{~mm} / \mathrm{h}$ ), while a positive concentration serum monoclonal anti-GBM antibody (isolated from bovine kidney as an antigen) was reported (258.3 EU/ml; cat no. EA 1251-9601 G, EUROIMMUN Medical Laboratory Diagnostics Co., Ltd, Luebeck, Germany). Serologic tests for antinuclear, anti-double-strand DNA and anti-neutrophil cytoplasmic antibodies were all negative. In addition, the serum levels of $\operatorname{IgA}$, IgG, IgM, complement C3 and $\mathrm{C} 4$, and total hemolytic complement (CH50) were within the normal ranges. Chest X-ray radiography (Ysio 50019, Siemens, Munich, Germany) was also normal, while ultrasonography (ACUSON S2000 Ultrasound system, Siemens, Chicago, IL, USA) showed normal shape of kidneys without kidney stones, and the sizes of the right and left kidneys were $102 \times 51$ and $101 \times 51 \mathrm{~mm}$ (the normal level is $90-110 \times 40-60 \mathrm{~mm}$ ), respectively. This can be due to the previous diagnosis being wrong or by the removal of the kidney stones with the previous treatment. Previous medical history of the patient revealed that the Scr was $57 \mu \mathrm{mol} / 1$ at 6 months prior to admission, while the patient had a 20-year history of recurrent tonsillitis. Based on the aforementioned findings, rapidly progressive glomerulonephritis was diagnosed and renal biopsy was immediately performed.

Light microscopy (DM2500, Leica) identified 18 glomeruli with 12 cellular crescents and 4 cellulofibrous crescents, and the glomeruli presented capillary occlusion with segmental fibrinoid necrosis (Fig. 1A). Numerous necrotic tubular epithelial cells and diffused infiltration of inflammatory cells indicated severe injury to the tubulointerstitium. The small arterial walls were thickened without significant evidence of necrosis or hyaline degeneration. Immunofluorescent examination (DM2500, Leica) revealed that strong IgG (3+) linear deposition in the capillary loop (Fig. 1B), along with mesangial staining for IgA (4+; Fig. 1C), IgM (1+) and C3 (2+). The staining intensity was scored as follows: $0+$, negative; 
1+, mild; $2+$, moderate or 3+, strong (8). Furthermore, electron microscopy (Hitachi 7700, Tokyo, Japan) demonstrated electron-dense mass deposition in the mesangium (Fig. 1D). Based on the aforementioned findings, the diagnosis of anti-GBM disease with IgA nephropathy was confirmed.

The patient was immediately administered $500 \mathrm{mg} /$ day intravenous methylprednisolone (Pfizer Ltd., Kent, UK) for 3 days, which was then changed to $60 \mathrm{mg} /$ day oral prednisone (Shanghai Xinyi Pharmaceutical Co., Ltd, Shanghai, China); however, the dosage was quickly decreased to $20 \mathrm{mg} /$ day two weeks later due to the side effect of hyperglycemia. Treatment with cyclophosphamide and plasmapheresis were then recommended, however this treatment was not accepted by the patient due to the possibility of side effects. Therefore, MMF (0.5 g, twice a day; Roche Pharmaceutical Co., Ltd, Shanghai, China) in combination with prednisone (20 mg/day) were administered to the patient and the dose of prednisone was decreased by $5 \mathrm{mg}$ every month. Four months later the prednisone treatment stopped. One month later gross hematuria was gradually relieved following MMF treatment. Further laboratory examinations after 3 months of MMF treatment showed the following results: Hematuria with 5-6 RBCs/HP; Scr level, $102 \mu \mathrm{mol} / \mathrm{l}$; Hb, $112 \mathrm{~g} / \mathrm{l}$; ESR, $22 \mathrm{~mm} / \mathrm{h}$; positivity for anti-GBM antibody $(176.0 \mathrm{EU} / \mathrm{ml})$. After 6 months, the $\mathrm{Scr}$ level was found to be $100 \mu \mathrm{mol} / \mathrm{l}$ and the ESR was $23 \mathrm{~mm} / \mathrm{h}$, showing no significant difference as compared with these levels 3 months before, whereas the $\mathrm{Hb}$ level was increased to $129 \mathrm{~g} / 1$ and the test for anti-GBM antibody was negative. The total MMF treatment was 15 months in total. Patient informed consent was obtained prior to the study.

In the last observation in February 2016 the MMF treatment had ceased and the laboratory examinations demonstrated the following results: Urine test showed $45 \mathrm{RBCs} / \mathrm{HP}$; Scr level, $74 \mu \mathrm{mol} / \mathrm{l} ; \mathrm{Hb}, 132 \mathrm{~g} / \mathrm{l}$; ESR, $4 \mathrm{~mm} / \mathrm{h}$ and the test for anti GBM antibody was negative.

\section{Discussion}

In the present study, a rare case of anti-GBM disease with IgA nephropathy was reported. Previous animal studies have demonstrated that complement activation through the classical complement pathway is one of the major mechanisms underlying glomerular injury in anti-GBM disease $(14,15)$. According to this proposed theory, complement Clq may serve an important role in the underlying mechanism of anti-GBM disease. However, a number of studies have reported that complement $\mathrm{C} 1 \mathrm{q}$ deposition was seldom present along the GBM in the renal tissue of patients, and circulating and urinary levels of C1q were not significantly correlated with the severity of kidney injury $(8,16)$. Hu et al (9) further found that, even in patients with $\mathrm{C} 1 \mathrm{q}$ deposition, no association of $\mathrm{Clq}$ with the disease activity and severity was identified, suggesting that the classical complement pathway may not serve a pathogenic role in the development of kidney injury in human anti-GBM disease. In the present case, immunofluorescence examination did not identify any C1q deposition, which is consistent with previous findings (9). However, the diagnosis of anti-GBM disease remains appropriate in the present case.

As the progression of anti-GBM disease can be rapid and the outcome is greatly associated with the severity at presentation, treatment must be initiated immediately. It is widely recognized that corticosteroids and cyclophosphamide in combination with plasmapheresis is the preferred standard treatment administered as the initial immunosuppressive therapy for anti-GBM disease (6). Notably, in the present study, the patient declined the standard treatment for anti-GBM disease, however a relatively improved therapeutic outcome with less side-effects was achieved compared with treatment with corticosteroids in combination with MMF. To date, there is little clinical evidence to recommend MMF for the anti-GBM disease therapy, although previous animal experiments in a rat model of anti-GBM disease have suggested the preventive effect of MMF against glomerular crescent formation (17). The case reported in the present study may provide useful evidence concerning the effect of MMF for the clinical treatment of anti-GBM disease.

As mentioned earlier, strong IgA deposition in the renal tissue may have a profound impact on the pathogenesis of this rare disease $(11,18)$. The findings from randomized controlled trials (RCTs) investigating MMF administration in IgA nephropathy are conflicting, since favorable outcomes of MMF have only been demonstrated in studies conducted in China (19-22). This is due to the fact that Asian IgG nephropathy patients may be more sensitive to MMF treatment, and the variation of ethnicity may be partly the reason for this observation. Based on the updated treatment options, the use of MMF is recommended for selected patients in whom steroid therapy has failed or who are intolerant to steroid therapy in IgA nephropathy (23). Nevertheless, MMF may prove to be a novel treatment for this rare disease, although the exact underlying mechanism requires further in-depth investigation.

In conclusion, the present study reported a case with anti-GBM disease and $\operatorname{IgA}$ nephropathy, in which the patient responded well to corticosteroids plus MMF treatment. The underlying mechanism renders further in-depth examination.

\section{References}

1. Lahmer T and Heemann U: Anti-glomerular basement membrane antibody disease: a rare autoimmune disorder affecting the kidney and the lung. Autoimmun Rev 12: 169-173, 2012.

2. Pedchenko V, Bondar O, Fogo AB, Vanacore R, Voziyan $P$, Kitching AR, Wieslander J, Kashtan C, Borza DB, Neilson EG, et al: Molecular architecture of the Goodpasture autoantigen in anti-GBM nephritis. N Engl J Med 363: 343-354, 2010.

3. Tang W, McDonald SP, Hawley CM, Badve SV, Boudville NC Brown FG, Clayton PA, Campbell SB, de Zoysa JR and Johnson DW: Anti-glomerular basement membrane antibody disease is an uncommon cause of end-stage renal disease. Kidney Int 83: 503-510, 2013.

4. Cui Z and Zhao MH. Advances in human antiglomerular basement membrane disease. Nat Rev Nephrol 7: 697-705, 2011.

5. Jennette JC and Nickeleit V: Anti-glomerular basement membrane glomerulonephritis and Goodpasture's syndrome. In: Heptinstall's Pathology of the Kidney. Jennette JC, Olson JL, Schwartz MM and Silva FG (eds). 6th edition. Lippincott Williams and Wilkins,Philadelphia, PA, pp 615-641, 2007.

6. Kidney Disease-Improving GlobalOutcomes Glomerulonephritis Work Group: KDIGO Clinical Practice Guideline for Glomerulonephritis. Kidney Inter Suppl 2: 139-274, 2012.

7. Hellmark T, Niles JL, Collins AB, McCluskey RT and Brunmark C: Comparison of anti-GBM antibodies in sera with or without ANCA. J Am Soc Nephrol 8: 376-385, 1997.

8. Fischer EG and Lager DJ: Anti-glomerular basement membrane glomerulonephritis: A morphologic study of 80 cases. Am J Clin Pathol 125: 445-450, 2006. 
9. Hu SY, Jia XY, Yang XW, Yu F, Cui Z and Zhao MH: Glomerular Clq deposition and serum anti-Clq antibodies in anti-glomerular basement membrane disease. BMC Immunol 14: 42, 2013

10. Gao B, Li M, Xia W, Wen Y and Qu Z: Rapidly progressive glomerulonephritis due to anti-glomerular basement membrane disease accompanied by $\operatorname{Ig}$ A nephropathy: A case report. Clin Nephrol 81: 138-141, 2014.

11. Wang A, Wang Y, Wang G, Zhou Z, Xun Z and Tan X: Mesangial IgA deposits indicate pathogenesis of anti-glomerular basement membrane disease. Mol Med Rep 5: 1212-1214, 2012.

12. Carreras L, Poveda R, Bas J, Mestre M, Rama I and Carrera M: Goodpasture syndrome during the course of a Schönlein-Henoch purpura. Am J Kidney Dis 39: E21, 2002.

13. Kielstein JT, Helmchen U, Netzer KO, Weber M, Haller H and Floege J: Conversion of Goodpasture's syndrome into membranous glomerulonephritis. Nephrol Dial Transplant 16: 2082-2085, 2001.

14. Otten MA, Groeneveld TW, Flierman R, Rastaldi MP, Trouw LA, Faber-Krol MC, Visser A, Essers MC, Claassens J, Verbeek JS, et al: Both complement and $\mathrm{IgG}$ fc receptors are required for development of attenuated antiglomerular basement membrane nephritis in mice. J Immunol 183: 3980-3988, 2009.

15. Sheerin NS, Springall T, Carroll MC, Hartley B and Sacks SH: Protection against anti-glomerular basement membrane (GBM)-mediated nephritis in C3-and C4-deficient mice. Clin Exp Immunol 110: 403-409, 1997.

16. Ma R, Cui Z, Liao YH and Zhao MH: Complement activation contributes to the injury and outcome of kidney in human anti-glomerular basement membrane disease. J Clin Immunol 33 172-178, 2013.
17. Takeda S, Takahashi M, Sado Y, Takeuchi K, Hakamata Y, Shimizu H, Kaneko T, Yamamoto H, Ito C, Ookawara S, et al: Prevention of glomerular crescent formation in glomerulonephritis by mycophenolate mofetil in rats. Nephrol Dial Transplant 19: 2228-2236, 2004.

18. Trpkov K, Abdulkareem F, Jim K and Solez K. Recurrence of anti-GBM antibody disease twelve years after transplantation associated with de novo IgA nephropathy. Clin Nephrol 49: 124-128, 1998.

19. Maes BD, Oyen R, Claes K, Evenepoel P, Kuypers D, Vanwalleghem J, Van Damme B and Vanrenterghem YF: Mycophenolate mofetil in IgA nephropathy: Results of a 3 -year prospective placebo-controlled randomized study. Kidney Int 65: 1842-1849, 2004.

20. Frisch G, Lin J, Rosenstock J, Markowitz G, D'Agati V, Radhakrishnan J, Preddie D, Crew J, Valeri A and Appel G: Mycophenolate mofetil (MMF) vs placebo in patients with moderately advanced IgA nephropathy: A double-blind randomized controlled trial. Nephrol Dial Transplant 20: 2139-2145, 2005.

21. Tang S, Leung JC, Chan LY, Lui YH, Tang CS, Kan $\mathrm{CH}$, Ho YW and Lai KN: Mycophenolate mofetil alleviates persistent proteinuria in IgA nephropathy. Kidney Int 68: 802-812, 2005.

22. Tang SC, Tang AW, Wong SS, Leung JC, Ho YW and Lai KN Long-term study of mycophenolate mofetil treatment in $\operatorname{Ig} \mathrm{A}$ nephropathy. Kidney Int 77: 543-549, 2010.

23. Hogan J, Mohan P and Appel GB: Diagnostic tests and treatment options in glomerular disease: 2014 update. Am J Kidney Dis 63: 656-666, 2014. 